\title{
Study of flow around model of cooling tower by means of 2D Particle Image Velocimetry measurement
}

\author{
Veronika Barraclough ${ }^{1}$, Jan Novotný ${ }^{2}$, and Pavel Šafaŕík ${ }^{2}$ \\ ${ }^{1}$ Aerospace Research and Test Establishment, PLC. (ARTE), Aerodynamics Department, Prague, Czech Republic \\ ${ }^{2}$ Czech Technical University in Prague, Faculty of Mechanical Engineering, Department of Fluid Dynamics and Thermodynamics, \\ Prague, Czech Republic
}

\begin{abstract}
This paper deals with flow around a bluff body of hyperboloid shape. It combines results gathered in the course of research by means of Particle Image Velocimetry (PIV). The experiments were carried out by means of low-frequency 2D PIV and the Reynolds number was 43000 .
\end{abstract}

\section{Introduction}

In this contribution, research in mechanics of fluids field, that developed from experimental testing for civil engineering, will be presented. Originally one of the well-known, and often investigated, problems of civil engineering - wind loading on cooling towers - had been investigated. There are plenty of articles dealing with this problem, whose enumeration can be found, for instance, in a summary article with a symptomatic name - A Survey on Hyperbolic Cooling Towers [1]. This paper is not going to deal with the cooling towers, it only originated there.

The object of measurement consisted of four models of hyperboloid cooling towers, whose centres were located in the corners of the square, and were tested in a wind tunnel designed for civil engineering experiments. According to the original measurement plans and requests from the industrial partner, the pressures inside and outside the model were measured and also calculated by means of mathematical modelling and numerical calculation. These two attitudes were compared and evaluated.

The experimental part described above was later extended to the pure mechanics of fluids discipline. The original test plan had determinated pressures on the models by classical means of fluid mechanics testing (pressure taps) which was completed by research of wakes with the help of a modern tool of non-intrusive measuring, Particle Image Velocimetry (PIV). This paper hopes to deal with some of the results gathered on this journey.

There are various paths the researcher can go by on the way to the results for the hyperboloid object. To get a comprehensive picture of flow it is possible to come out of the mean flow topology of the cylinder, as depicted for instance in [2], page 14, Picture 2. Therefore, in flow around the hyperboloid object we could expect horseshoe vortex, tip vortex, arch vortex and trailing vortex and some clues of these patterns were indicated in the presented measurement. The aim of this work is to test possibilities of low frequency 2D PIV for the measurement of 3D structures of flow field and to identify areas of later research by means of hot wire anemometry and numerical calculation.

\section{Experimental Facility}

The experimental part accomplished so far was provided by means of 2D Particle Image Velocimetry (PIV), being the quickest tool for instant viewing of the problem. The measurement was provided in a range of Reynolds numbers varying from 43000 up to 130000; this contribution contains results for $\mathrm{Re}=43000$. The Reynolds numbers varied by the change of free stream velocity $U_{\infty}$ in the wind tunnel. The tunnel used for the measurement was the Boundary Layer Wind Tunnel (BLWT). It is equipped with a special surface helping the development of the boundary layer, so that the intensity in the main measured plane reaches $13 \%$ [3]. The ratio of the height of the model to the thickness of the modelled boundary layer in the BLWT is $\frac{1}{3}$. The BLWT dimensions of the rectangular measurement area are $1,8 \times 1,5$ metres.

The scale of the measured model of the cooling tower was $1: 400$. The model was printed using a $3 \mathrm{D}$ printer as $0,33 \mathrm{~m}$ tall plastic object. 


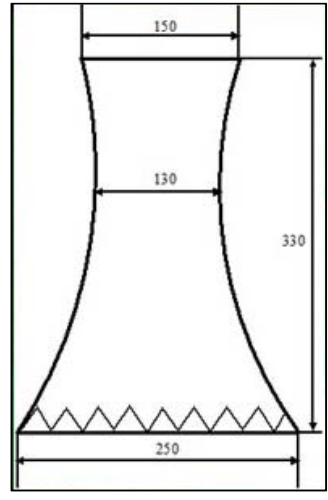

Fig. 1. Model of hyperboloid-shape cooling tower and its dimensions.

The PIV experiment was provided in a classical way - the measurement area was seeded with olive oil droplets produced by Laskin nozzles and a green laser light was used to illuminate the area. The main 2D experimental part at the beginning was provided with a laser with Nd:YAG crystals and low frequency $(15 \mathrm{~Hz})$ of the pulses.

The main characteristics of PIV measurements:

\begin{tabular}{|l|l|}
\hline PIV method used: & $2 \mathrm{D}$ \\
\hline Max. in-plane velocity: & $5 \mathrm{~m} / \mathrm{s}$ \\
\hline Max. cross-plane vel.: & $5 \mathrm{~m} / \mathrm{s}$ \\
\hline Field of view: & See Results \\
\hline Interrogation area: & $32 \times 32$ pixels \\
\hline Observation distance: & Maximum $1000 \mathrm{~mm}$ \\
\hline Recording method: & double-frame, single expos. \\
\hline Recording medium: & $\begin{array}{l}\text { CMOS sensor, } 1280 \times 1024 \\
\text { pxl, pixel size } 12 \times 12 \mu \mathrm{m}\end{array}$ \\
\hline Recording lens: & $\begin{array}{l}\text { FL }=50 \mathrm{~mm}, 35 \mathrm{~mm} \text { for } \\
\text { details, f } \#=1,2-2\end{array}$ \\
\hline Illumination: & YAG laser, 2x $60 \mathrm{~mJ} /$ pulse \\
\hline Pulse delay: & $100 \mu \mathrm{s}$ \\
\hline Recording frequency: & $15 \mathrm{~Hz}$ \\
\hline
\end{tabular}

For data evaluation, Dynamic Studio software was used. All image processing, and later calculations of particle movements in two consequentive images were provided with the help of this post-processing tool. For this work, adaptive correlation was used with these parameters: final interrogation area was 32 × 32 pixels, which represented 2.3 to 6.5 times 1.9 to 6.3 square millimetres of area. Horizontal and vertical overlap were $50 \%$. Evaluated vectors were filtered by range validation and universal outlier detection. Usually, the vector statistics representing averaged velocity field was calculated.

\section{Measurements and results}

\subsection{Incoming flow}

Measurements were carried out in front of the object, on its side and in its wake. In the case of the front part, the measurement plane was set to the axis of the model and parallel to the flow, perpendicular to the ground. Note the small icons in the velocity fields for positioning of the illuminated area. There were vortices observed in instant velocity fields nearby the bottom of the object (Fig. 2) and the velocity component extracts show some movement against the flow direction (Fig. 3). The averaged velocity field (Fig. 4) doesn't indicate the presence of the structure, which would encircle the bottom part of the object, though. The cause of it could apparently consist of the airway of the bottom part of the model, or could be the result of an inappropriate scale of the measurement. This area will definitely be a subject of later, more detailed research.

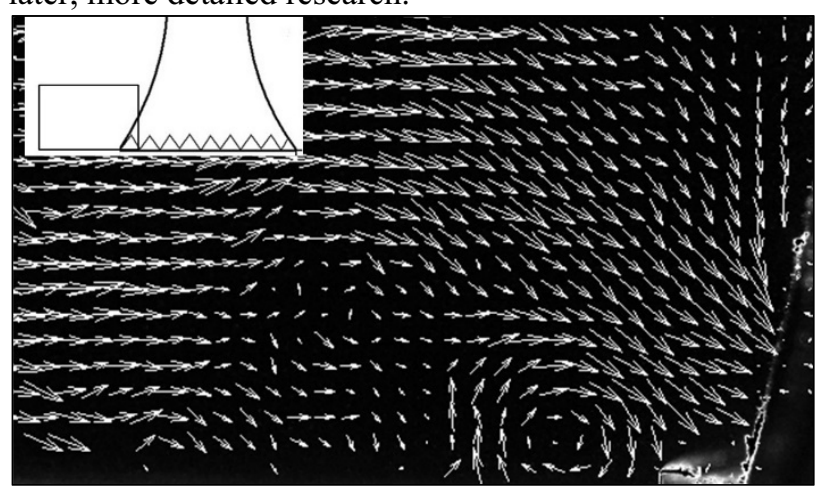

Fig. 2. Instant velocity field of the front bottom part of the model. Parallel to the flow, perpendicular to the ground. The field of view $94 \times 62 \mathrm{~mm}$.

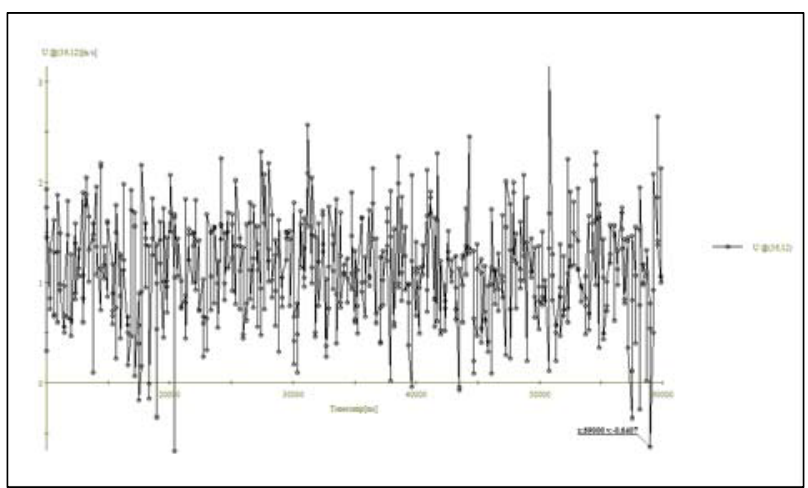

Fig. 3. Velocity x-component development in time. The extract was made in the front of the model and close to the ground (in the centre of the vortex depicted on Fig.2).

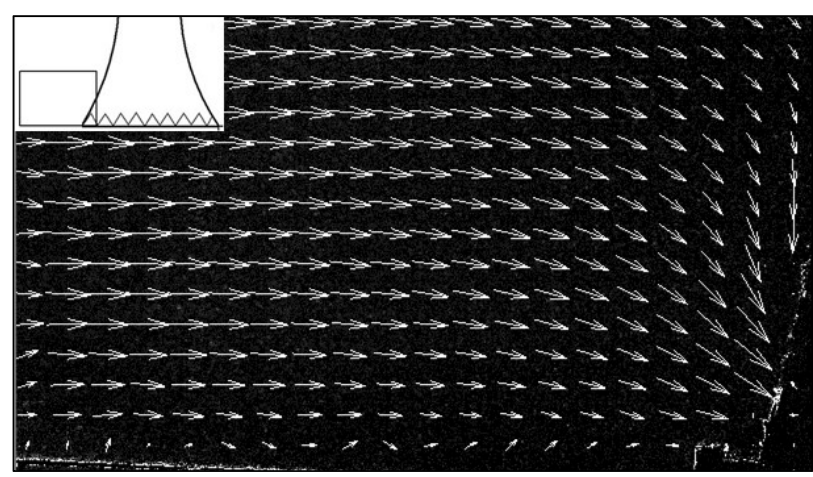

Fig. 4. Average velocity field of the front bottom part of the model. Parallel to the flow, perpendicular to the ground. The field of view 94 x $62 \mathrm{~mm}$. 


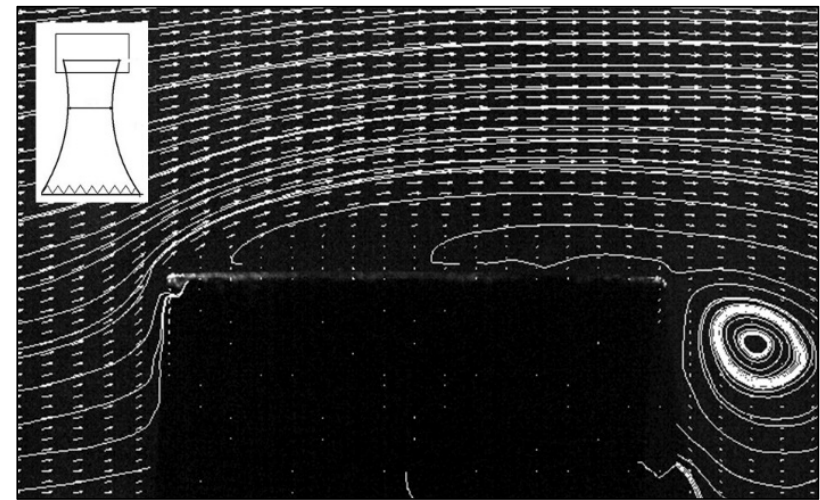

Fig. 5. Averaged velocity field and streamlines around the top of the object. Parallel to the flow, perpendicular to the ground. The field of view $260 \times 208 \mathrm{~mm}$.

\subsection{The wake}

There was special attention paid to the measurement in the wake. The aim of the first part of the measurement was to compare the influence of the turbulence intensity on a wake character, so two types of wind tunnels with different rates of turbulence were used. The evaluation of this measurement is not part of the paper.

Measurement planes for the wake were parallel to the flow direction in the object axis and perpendicular to it. For the case of measurement in the object axis, the whole picture was put together from two positions of the camera. This solution was chosen due to lack of space in the measurement area of BLWT for all equipment needed.
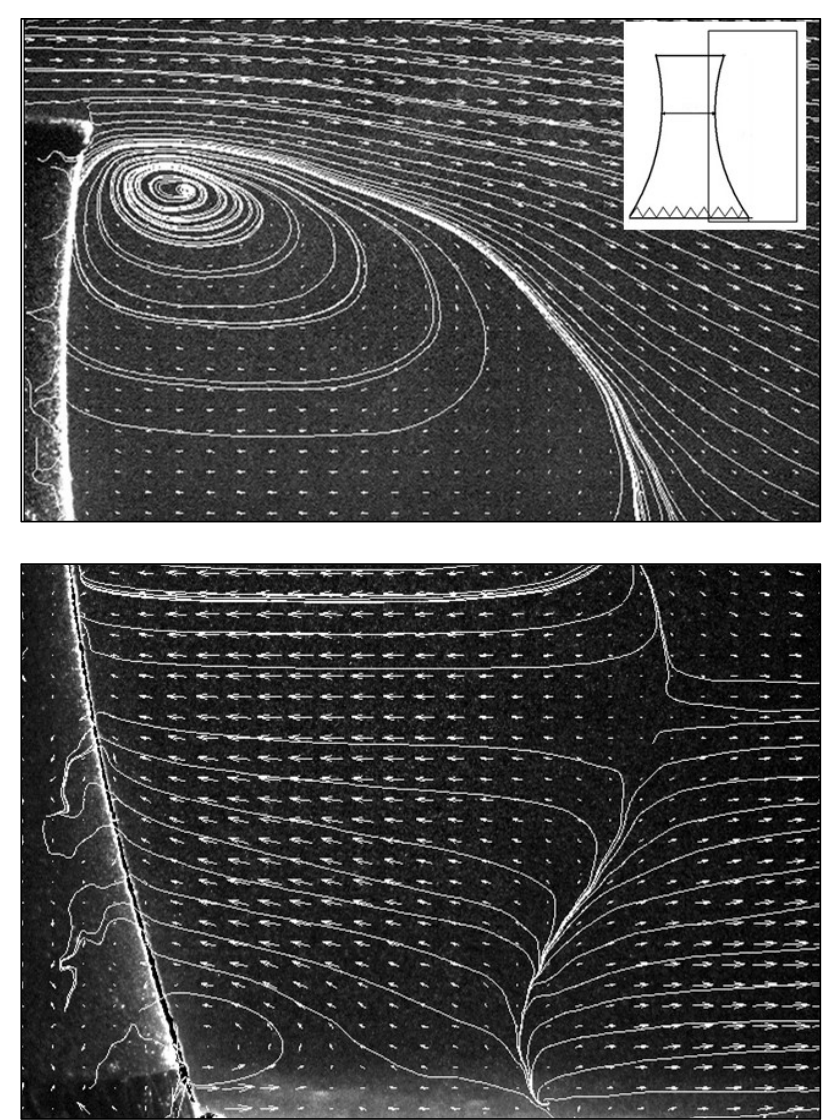

Fig. 6. Averaged velocity field and streamlines behind the model. The plane parallel to the flow, perpendicular to ground. The field of view $286 \times 229 \mathrm{~mm}$.

The view into the wake, the perpendicular plane was supposed to offer some proof of existence of the typical 3D flow structures. It was later realized that the PIV measurement near the ground of BLWT, covered with special material to induce natural-like conditions, is very difficult due to strong light reflection from this rough, uneven surface. This problem leads to some captured data being worthless and needs to be solved more carefully in future with other tools of experimental fluid mechanics.

There were measurements in two planes in different distances from the bottom of the model provided and several characteristics evaluated (Fig. 7,8, in this paper only more interesting data from the plane closer to the model are introduced).

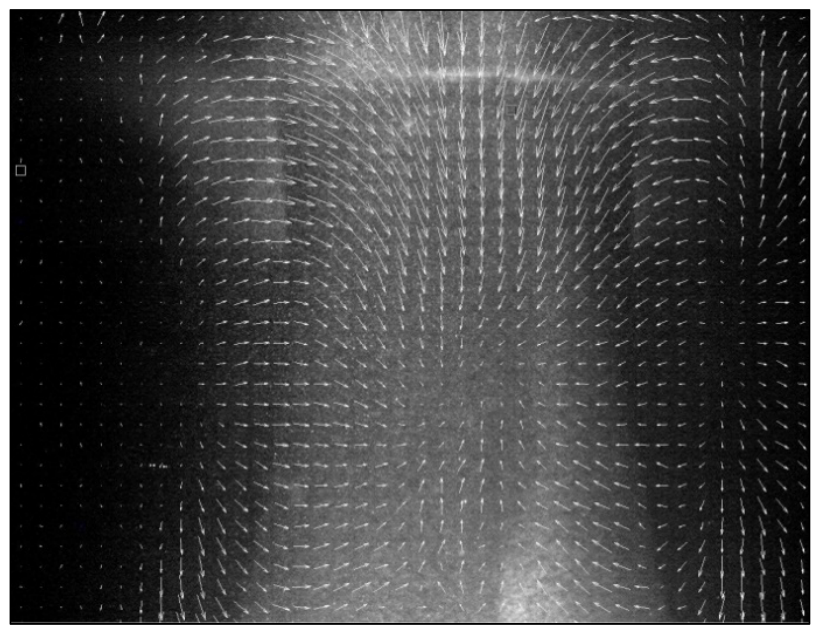

Fig. 7. Averaged velocity field, distance $56 \mathrm{~mm}$ from the bottom of the model. The plane perpendicular to the flow and the ground. The field of view $286 \times 229 \mathrm{~mm}$.

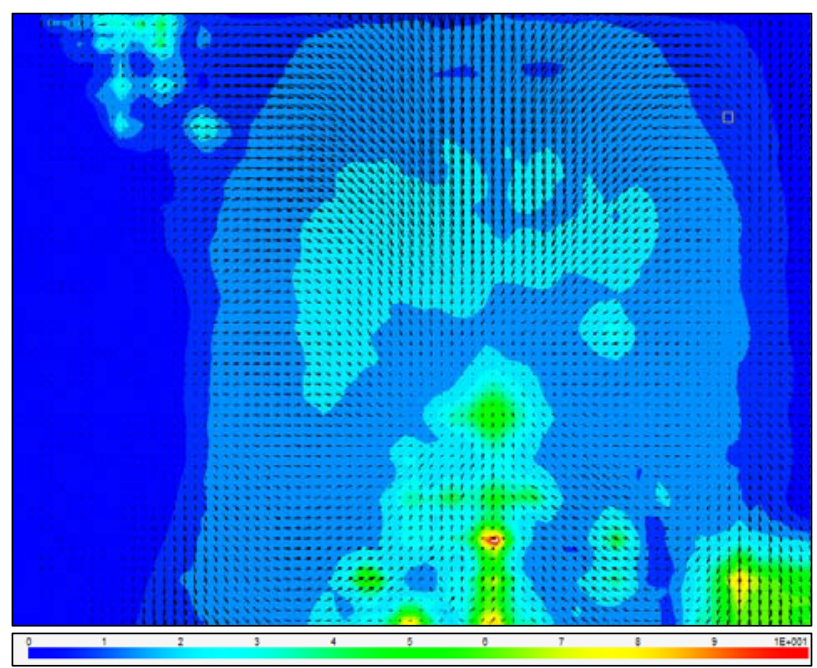

Fig. 8. Sum of variances, which is proportional to turbulent kinetic energy in units $\left(\left(\frac{m}{s}\right)^{2}\right)$. The plane perpendicular to the flow direction and to the ground. The distance $56 \mathrm{~mm}$ from the bottom of the model. 
It has to be emphasized here, that the software evaluates the velocities from a spatial average over the interrogation area, while the classical attitude would be infinitely small elements. Therefore, the Fig. 8 shall serve for later comparison to numerical calculation.

And in the end, the measurement in the plane parallel to the ground where the object has the narrowest diameter shouldn't be omitted. The measuring plane was set at an altitude of $0.2 \mathrm{~m}$ from the ground desk.

As expected, the averaged velocity field and the streamlines show two symmetric eddies. Dimensionless recirculation length in this case is less than 1; such limited recirculation area is caused by turbulence in the free stream supressing the wake. The shape of the wake shows turbulent separation with the separation point behind the widest area of the model.

For later research, this part could be even more interesting: according to [4], shedding of vortices changes from Kármán asymmetric type to symmetric arch type at a geometrical aspect ratio of an object (cylinder) of 2.5; as the aspect ratio of our measured object varies from 1.3 to 2.54 , the narrowest area of it could be measured with respect to this fact.

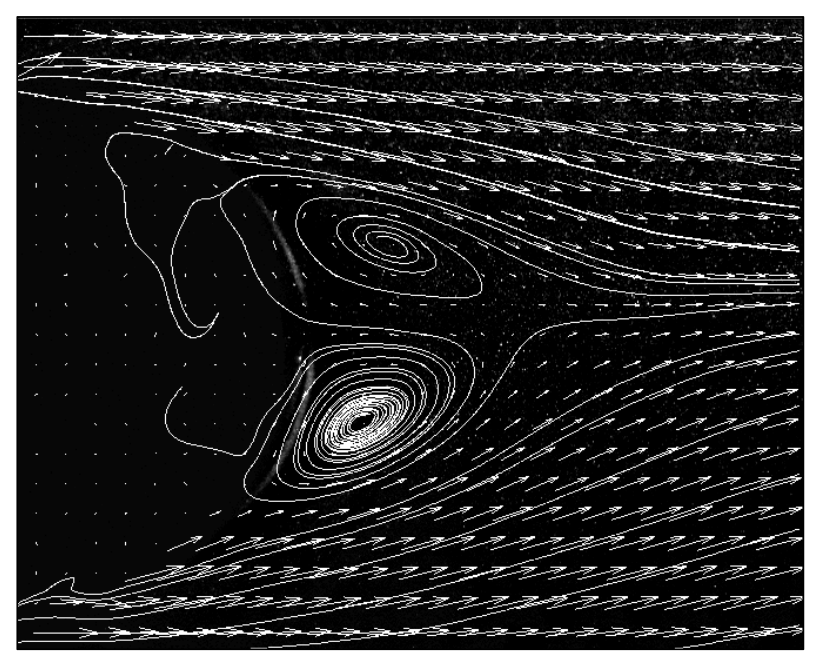

Fig. 9. Averaged velocity field and streamlines in the wake, plane parallel to the ground. The field of view $250 \times 200 \mathrm{~mm}$.

\section{Conclusions}

The model of hyperboloid shaped object was measured by means of 2D Particle Image Velocimetry in the Wind Tunnel with simulated atmospheric boundary layer. The model was tested in subcritical range of Reynolds numbers and results from it were presented. Various planes around the model were captured: in the front of the model, the plane parallel to the flow and perpendicular to the ground was measured and a more detailed view to the flow was obtained as well, with the extraction of velocity components in small distances from the object bottom. The bottom part of the model consists of pillars and is penetrable for the flow, which might influence the formation of horseshoe vortex. The measurement didn't show this structure, which might be caused by the inappropriate scale of the view or bad placing of the capturing camera. The model is hollow, so the suction effect of this part will somehow influence an outfall and the flow around it.

There were several planes measured on the side of the model as well. All planes were parallel to the flow, perpendicular to the ground. The strong 3D character of the flow requests the information of third velocity component or at least other measurements of the same kind, but in the plane perpendicular to the flow direction.

Special care was dedicated to the wake. There were three planes chosen to be measured. One was set in the model axis and perpendicular to the ground. The averaged velocity field of this measurement revealed a possible arch vortex behind the model or highlighted point of later research. Next, the plane perpendicular to the ground was measured. As expected, the averaged velocity field showed two symmetrical formations. And eventually, the plane perpendicular to both flow direction and the ground completed the measurement in the wake. Instant velocity fields were used for testing of vorticity identification tools, but all these tools used in the software calculated with strong uncertainties and didn't show us any useful results. The average velocity field served for calculation of sum of variances proportional to turbulent kinetic energy in units.

In conclusion, it could be said that from all the $2 \mathrm{D}$ PIV measurements considering spatial formations around such a bluff body raised more questions than answers.

The research was performed with financial institutional support from government budget through the Ministry of Industry and Trade of the Czech Republic.

The support from the Technology Agency of the Czech Republic under Project No. TE 01020036 is gratefully acknowledged.

\section{References}

[1] E. Asadzadeh, M. Alam, A Survey on Hyperbolic Cooling Towers, Intern. Journal of Civil, Envir., Struct., Construct. and Arch. Engineering 8, (2014)

[2] R. J. Pattenden, S. R. Turnock, X. Zhang, Measurements of the flow over a low-aspect-ratio cylinder mounted on a ground plane, Experiments in Fluids 39,10-21(2005)

[3] P. Michálek, D. Zacho, R-6123 Report, ARTE, Plc, Prague, Czech Republic (2014)

[4] H. Sakamoto, M. Arie Vortex shedding from a rectangular prism and a circular cylinder placed vertically in a turbulent boundary layer. J Fluid Mech 126, 147-165 (1983)

[5] N. P. van Hinsberg, J.-M. Lueckhof, J. Nuhn, The Reynolds number effect from subcritical to high transcritical on steady and unsteady loading on a rough circular cylinder, Proceedings of the 9th Intern. Confer. on Struct. Dyn., EURODYN (2014)

[6] X. P. Liu, L. Zhao, Y. J. Ge, Extreme value distribution of surface aerodynamic pressure of hyperbolic cooling tower, The 7th Inter. Colloq. on Bluff Body Aerodynamics and Applications (BBAA7) Shanghai, China; (2012) 
[7] A. Roshko, On the development of turbulent wakes from vortex streets, NACA Report 1191 (1954)

[8] P. Chakraborty, S. Balachandar, R. J. Adrian, Kinematics of Local Vortex Identification Criteria, Journal of Visualization, Vol. 10, No. 2 137-140, (2007)
[9] V. Holmén, Methods for Vortex Identification, Lund University Libraries,

lup.lub.lu.se/student-papers/record/3241710 (2012)

[10] M. Jirsák, Physical Principles of Work with Boundary Layer Wind Tunnel, ARTE Plc., Prague, Czech

Republic

(2012) 